\title{
An Efficient Blood Vessel Segmentation from Color Fundus Image
}

\author{
Sachin Sridhar \\ NIT Puducherry \\ Karaikal, India
}

\author{
K. J. D. S Srinivas \\ Rao \\ NIT Puducherry \\ Karaikal, India
}

\author{
N. Hemanth \\ NIT Puducherry \\ Karaikal, India
}

\author{
Malaya Kumar Nath \\ NIT Puducherry \\ Karaikal, India
}

\begin{abstract}
Retinal vessel segmentation and delineation of morphological attributes of retinal blood vessels are utilized for diagnosis, screening and evaluation of various ophthalmologic diseases. In this paper, local entropy-based thresholding and modified Gaussian-based matched filter segmentation techniques have been performed on the bit plane sliced images to assess the information of blood vessels present in different bits. Bit plane slicing has been used prior to thresholding for efficient segmentation and further processing as it highlights the contribution made to the total image appearance by specific bits. This will help in efficient transmission of retinal data and diagnosis of diseases in retinal images. The efficiency of the segmentation method is calculated by evaluating performance measures. This is useful for image compression, robust person identification and efficient transmission. Local entropy-based thresholding is used on the reconstructed image, obtained by combining specific bits in bit plane slicing, for segmentation. This method is tested on the publicly available DRIVE and Aria databases. Performance measures are calculated for the segmentation methods. Supervised segmentation on bit planes sliced images performs better than Gaussian matched filter method.
\end{abstract}

\section{General Terms}

Image segmentation, compression et. al.

\section{Keywords}

Bit plane slicing, local entropy-based segmentation, matched filter, specificity

\section{INTRODUCTION}

Retina is responsible for vision and affected by diabetic retinopathy and leads to vision loss. At the initial stage of diabetic retinopathy blood vessels are affected. The morphological attributes of blood vessels are used for diagnosis and are captured by fundus camera [1]. The segmentation of the retinal image structure has been of great interest as it could be used as an aid in the diagnosis of ophthalmology. Retinal blood vessel segmentation is useful in detecting various retinal diseases, as well as person identification. Every individual has a unique orientation of blood vessels across the optic disc. This is the basis of person identification [2, 3]. Segmentation of blood vessels is done manually by expert ophthalmologists, but this is a time consuming task and prone to human errors. Therefore, automatic detection of blood vessels is necessary for efficient transmission and person identification. Blood vessel segmentation methods discussed in the literature [1] are pattern recognition techniques, matched filter techniques, vessel tracking/tracing, mathematical morphology, multiscale approaches and model-based approaches. Modified matched filter with double-sided thresholding has been used by Zhang et al., [4] to enhance the blood vessels in retinal fundus image. Pang et al. used mathematical morphologybased bottom hat transformation [5] for the extraction of blood vessels. These methods cannot be used directly for efficient transmission as well as person identification. Hence, bit plane slicing is used before performing blood vessel segmentation. In bit plane slicing, the pixels present in the digital image can be expressed in terms of bits [6]. Specific bits are highlighted to obtain the maximum blood vessel information in the color fundus image. This helps in representing the blood vessels in compressed form. The segmentation algorithms have been applied to the reconstructed image obtained by considering the selective bits in bit plane slicing.

The rest of the paper is organized as follows. The proposed method is discussed in Section 2. Results and discussions are discussed in Section 3 and the conclusions are stated in Section 4.

\section{METHODOLOGY}

The proposed method for efficient blood vessel segmentation for transmission and person identification has been given in Figure. 1. It consists of pre-processing, bit plane slicing, segmentation by Gaussian-based matched filter method and local entropy-based thresholding, followed by evaluation of performance measures. Depending on the performance measures and the mean opinion score, the bits highlighting the blood vessel information have been selected for transmission and person identification.

\subsection{Pre-Processing}

The green channel of the color fundus image is used for further processing, since it has a better contrast as compared to the red and blue channels. The green channel image is resized to the form $2^{\mathrm{N}} \times 2^{\mathrm{N}}$. In this case, the value of $\mathrm{N}$ is equal to 9 . This is subjected to histogram equalization for uniform distribution of energy.

\subsection{Bit plane Slicing}

The pre-processed image is subjected to bit plane slicing. The contribution of individual bit planes to the blood vessels present in the fundus image has been analyzed. Individual bit planes are selectively combined based on the blood vessel information contained in each bit plane. The bits with more information have been considered and the bits with lesser information have been rejected. It has been found that bit plane ' $\mathrm{C} 5$ ' contains maximum information and bit plane ' $\mathrm{C} 0$ ' contains the least information. 


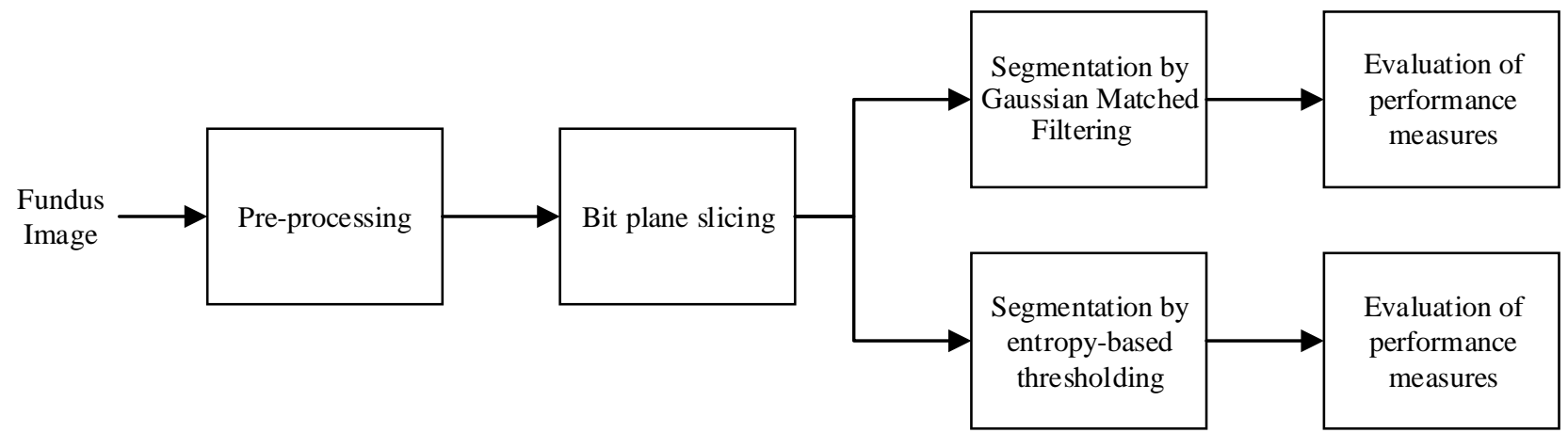

Figure 1. Block diagram for the proposed method

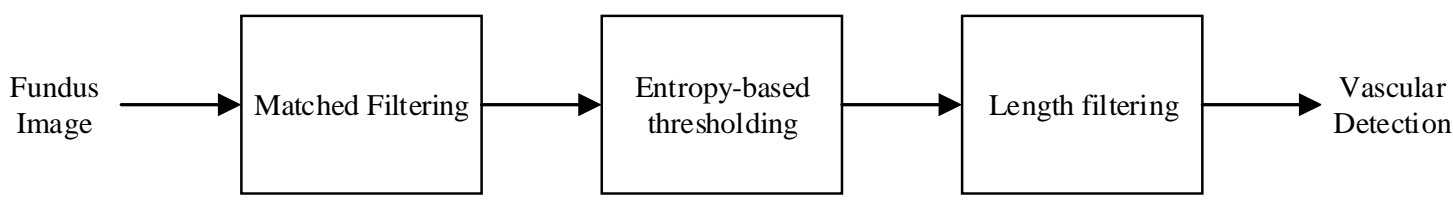

Figure 2. Block diagram describing the process of local entropy - based thresholding

\subsection{Blood Vessel Segmentation}

In this paper, Gaussian-based matched filter segmentation and local entropy based thresholding are used for the segmentation of the blood vessels from the bit plane sliced image. Local entropy-based thresholding consists of matched filtering, local entropy-based thresholding, length filtering and vasculature intersection detection [7]. The different steps for this method are given in Figure. 2. The pixel value of the cross section of the blood vessels takes the shape of a Gaussian curve, so a matched filter is used to match the blood vessels. It basically enhances the blood vessels [8]. Entropy-based thresholding is performed to retain the spatial structure of the vascular segments. Length filtering is used to remove misclassified or isolated pixels, using the concept of connected pixels labeling [9]. An efficient local entropy-based segmentation algorithm has been used, which considers the spatial distribution of gray levels. First, a co-occurrence matrix of the image has been defined to represent the transition of intensities [10].

$\mathrm{T}=\left[t_{i j}\right]$ and $t_{i j}$ is defined as

$t_{i j}=\sum_{l=1}^{P} \sum_{k=1}^{Q} \delta$

Where $\delta=1$ for co-occurring pixels and 0 otherwise. Considering a threshold ' $\mathrm{v}$ ', such that $0<\mathrm{v}<\mathrm{L}-1$, where $\mathrm{L}$ is the length of an individual blood vessel segment. In this case, ' $\mathrm{v}$ ' can partition the co-occurrence matrix into four quadrants, namely W, X, Y and Z, which are shown in Figure. 3. The probability of finding a pixel in region ' $\mathrm{W}$ ' can be defined as

$P_{W}=\sum_{i=0}^{v} \sum_{j=0}^{v} p_{i j}$

Similarly, the probability of finding a pixel in region ' $\mathrm{Y}$ ' is defined as

$P_{Y}=\sum_{i=v+1}^{L-1} \sum_{j=v+1}^{L-1} p_{i j}$

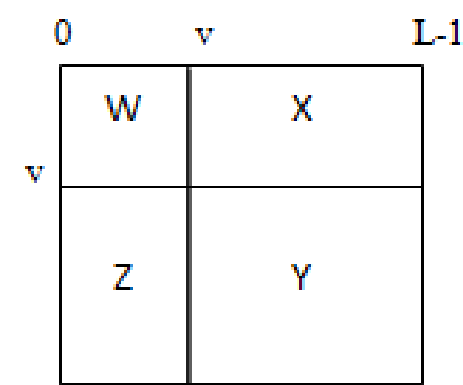

L-1

Figure 3. Partitioning the co-occurrence matrix

The probabilities within each individual quadrant have been normalized, such that the sum of the probabilities of the quadrants becomes equal to 1 .

$p_{i j}{ }^{W}=\frac{t_{i j}}{\sum_{i=0}^{v} \sum_{j=0}^{v} t_{i j}}$

Similarly, the normalized probability of quadrant $\mathrm{Y}$ is

$p_{i j}^{Y}=\frac{t_{i j}}{\sum_{i=v+1}^{L-1} \sum_{j=v+1}^{L-1} t_{i j}}$

The second order entropy of the object can be defined as

$H_{W}^{(2)}(s)=-\frac{1}{2} \sum_{i=0}^{v} \sum_{j=0}^{v} p_{i j}{ }^{W} \log _{2} p_{i j}{ }^{W}$

The second order entropy of the background is written as $H_{Y}^{(2)}(s)=-\frac{1}{2} \sum_{i=v+1}^{L-1} \sum_{j=v+1}^{L-1} p_{i j}{ }^{Y} \log _{2} p_{i j}{ }^{Y}$ 
Table 1: Comparison of performance measures of the two segmentation techniques

\begin{tabular}{|c|c|c|c|c|c|c|c|c|c|c|}
\hline \multicolumn{9}{|c|}{ Performance Measures } \\
\hline \multicolumn{7}{|c|}{ Gaussian Matched Filtering } & \multicolumn{5}{c|}{ Supervised segmentation } \\
\hline & TEST_M1 & TEST_M2 & TRAINING & ARIA_A & ARIA_D & TEST_M1 & TEST_M2 & TRAINING & ARIA_A & ARIA_D \\
\hline TP & 10721.7 & 9348.8 & 9366.6 & 13011.2 & 15759.4 & 18135.7 & 13223.4 & 16764.7 & 25292.7 & 27559.9 \\
\hline TN & 220844.85 & 230438.6 & 219402.8 & 197319.6 & 189920.8 & 217074.35 & 223128.7 & 218055.1 & 171161.1 & 174441.7 \\
\hline FN & 7984.8 & 9357.7 & 10129.4 & 16679.3 & 14991.3 & 11755.3 & 16667.6 & 11477 & 42837.8 & 30470.4 \\
\hline SPF & 90.7 & 94.7 & 90.4 & 84.9 & 82.1 & 93.7 & 96.2 & 93.5 & 88.6 & 85.8 \\
\hline SEN & 9.3 & 5.3 & 9.6 & 15.1 & 17.9 & 6.3 & 3.8 & 6.5 & 11.4 & 14.2 \\
\hline ACC & 88.3 & 91.5 & 87.3 & 80.2 & 78.5 & 89.7 & 90.2 & 89.6 & 74.9 & 77.1 \\
\hline EER & 5.8 & 4.3 & 6.4 & 9.9 & 10.8 & 5.1 & 4.9 & 5.2 & 12.5 & 11.5 \\
\hline KC & -270.7 & -256.3 & -388.6 & -344.9 & -275.9 & -210.2 & -210.8 & 6459.8 & -223 & -171.1 \\
\hline KJ & 26.9 & 30.9 & 23.3 & 19.8 & 21.8 & 38.3 & 32.2 & 35.2 & 28.9 & 31.1 \\
\hline KD & 41.5 & 46 & 36.4 & 32.8 & 35.6 & 53.7 & 47.7 & 49.6 & 43.8 & 46.6 \\
\hline
\end{tabular}

The total second order entropy of the object and the background can be written as:

$$
H_{T}^{(2)}(s)=H_{W}^{(2)}(s)+H_{Y}^{(2)}(s)
$$

The gray level corresponding to the maximum second order entropy gives the optimal threshold for object-background classification. Here, length filtering is used to remove isolated or misclassified pixels, using the concept of connected pixels labeling.

\subsection{Evaluation of Performance Measures}

In order to test the efficiency of the two segmentation methods, various performance measures such as True Positive, True Negative, Specificity, Sensibility, Accuracy, Equal Error Rate, Dice coefficient and Jaccord coefficient have been evaluated and compared [1]. From the mean opinion score and the performance measures, it may be concluded that segmentation by supervised local entropybased thresholding on bit plane sliced image outperforms the Gaussian-based matched filter method.

\section{RESULTS AND DISCUSSIONS}

Analysis of the segmentation techniques has been performed on the publicly available DRIVE [4] and Aria databases [11]. The DRIVE database comprises two sets of 20 images eachnamely Test and Training. DRIVE consists of segmented images in two manuals, namely Manual 1 and Manual 2. Analysis on the images of the Aria database has been applied on Aria A and Aria D. In bit plane slicing, bit 'C5' has been found to contain maximum blood vessel information, followed by 'C4', 'C6' and ' $\mathrm{C} 3$ '. These individual bits have been selectively combined to generate ' $\mathrm{C} 3456$ ', which contains majority of the blood vessel information. Figure 4 shows the blood vessel information contained in the individual bit planes.

Figure.4.(a) shows the extracted blood vessels marked by the experts. Figure.4.(b) to Figure. 4.(i) show the blood vessel information contained in bit plane $\mathrm{C} 0$ to $\mathrm{C} 7$ respectively. In these cases, the information in only one bit plane is present, as all the other bit planes have been zeroed. Figure.4.(j) shows the information contained in the combination of bit planes $\mathrm{C} 4$ and $\mathrm{C} 5$, zeroing all other bit planes. Figure.4.(k) shows the information contained in the combination of bit planes $\mathrm{C} 3, \mathrm{C} 4$ and $\mathrm{C}$, zeroing all other bit planes. Figure.4.(1) shows the information contained in the combination of bit planes $\mathrm{C} 3$, C4, C5 and C6, zeroing all other bit planes.

The extracted blood vessels in different planes are compared with the manually segmented blood vessels by the experts and tabulated in Table 1. DRIVE Test contains two manually segmented images, manual-1 (M1) and manual-2 (M2). Test M1 and Test M2 represent the performance measures of segmented blood vessels compared with M1 and M2 respectively. In Table 1 , true positive (TP), true negative (TN), false negative (FN), specificity (SPF), sensibility (SEN), accuracy (ACC), equal error rate (EER), conformity coefficient (KC), Jaccord coefficient (KJ) and Dice Coefficient (KD) are calculated for Gaussian-based matched filter and local entropy-based blood vessel segmentation. From the table, it is found that true positive, true negative, specificity, accuracy, KJ and KD are higher for local entropybased supervised segmentation. From the performance measures, it may be concluded that local entropy-based supervised segmentation of blood vessels outperforms Gaussian matched filtering based segmentation.

In Figure 3, the blood vessels have been considered in quadrant $\mathrm{W}$ and the background has been considered in quadrant $\mathrm{Y}$, which gives a high contrast. We may also consider the blood vessels in quadrant $\mathrm{Z}$ with the background in quadrant $\mathrm{Y}$ and obtain robust results. The analysis fails for all other selections of the quadrants. 


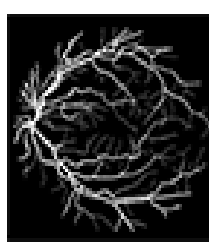

(a)

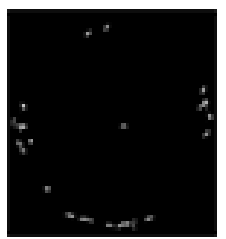

(d)

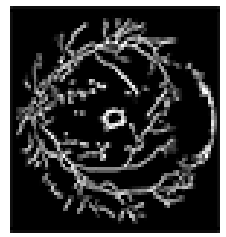

(g)

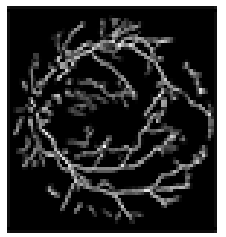

(j)

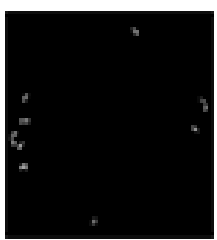

(b)

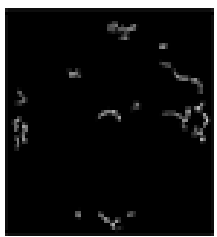

(e)

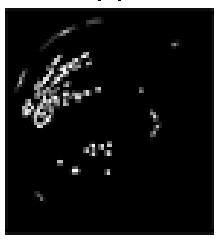

(h)

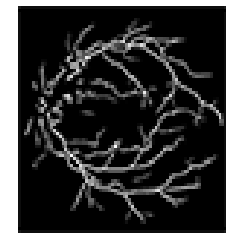

(k)

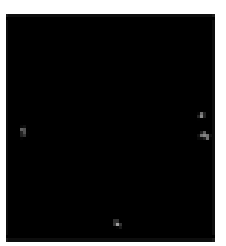

(c)

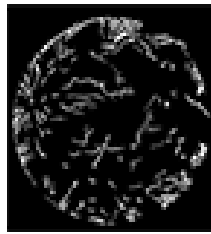

(†)

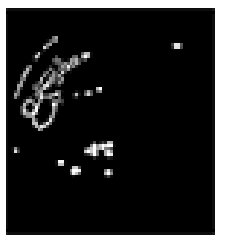

(i)

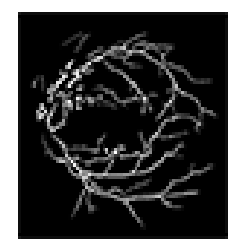

(1)
Figure 4. Blood vessel information contained in different bit planes

\section{CONCLUSION}

In this paper, the contribution of blood vessel information by the individual bit planes has been studied. Bit plane ' $\mathrm{C} 5$ ', contains maximum blood vessel information, followed by 'C4', 'C6' and 'C3'. These bit planes have been selectively combined to generate image ' $\mathrm{C} 3456$ ', which contains the majority of the blood vessel information. The other bit planes which contain lesser information have been rejected. The Local entropy-based thresholding segmentation has been applied to the selectively combined bit planes of the color fundus image. This method has been compared with the Gaussian-based matched filter segmentation technique. From the evaluated performance measures and the mean opinion score, it may be concluded that the supervised local entropybased segmentation algorithm outperforms the Gaussianbased matched filter method.

Blood vessel pattern is the unique feature at the optic disc and can be used for person identification. Blood vessel segmentation on bit planes reduces the coefficients for computation. This helps efficient transmission and storage of blood vessels.

\section{REFERENCES}

[1] M. M. Fraza, P. Remagninoa, A. Hoppea, B. Uyyanonvarab, A. R. Rudnickac, C.G. Owenc, and S.A. Barmana, "Blood vessel segmentation methodologies in retinal images - A survey", Computer Methods and Programs in Biomedicine, vol.108, pp. 407-433, 2012.

[2] Keisuke Fukuta, Toshiaki Nakagawa, Yoshinori Hayashi, Yuji Hatanaka, Takeshi Hara, and Hiroshi Fujita, "Personal identification based on blood vessels of retinal fundus images", Proc. of SPIE, vol. 6914, 2008.

[3] Cemal Kose, and Cevat Ikibas, "A personal identification system using retinal vasculature in retinal fundus images", Expert Systems with Applications, vol. 38, pp. 13670-13681, 2011.

[4] L. Zhang, Q. Li, J. You, and D. Zhang, "A modified matched filter with double sided thresholding for screening proliferative diabetic retinopathy," IEEE Transactions on Information Technology in Biomedicine, vol. 13, no. 4, pp. 528-534, July 2009.

[5] Kee Yong Pang, Lila Iznita I, M. H. Ahmad Fadzil, A. N. Hanung, N. Hermawan, and S. A. Vijanth, "Segmentation of retinal vasculature in color fundus images."Innovative Technologies in Intelligent Systems and Industrial Applications, Monash University, Sunway Campus, Malaysia: IEEE, July 2009, pp. 398-401.

[6] R. C. Gonzalez, and R. E. Woods, Digital Image Processing, 3rd ed., Pearson Prentice Hall, Upper Saddle River, NJ, 2008, pp. 477-501, Chap. 7.

[7] N. Patton, T. Aslam, T. MacGillivray, A. Pattie, I. J. Deary, and B. Dhillon, "Retinal vasculature image analysis as a potential screening tool for cerebrovascular disease: a rationale based on homology between cerebral and retinal microvasculatures," Journal of Anatomy, vol. 4, pp. 319-348, 2005.

[8] H. Li, W. Hsu, M. L. Lee, and T. Y. Wong, "Automatic grading of retinal vessel caliber," IEEE Transactions on Biomedical Engineering, vol. 52, no. 7, pp. 1352-1355, 2005.

[9] M. Niemeijer, J.J. Staal, B. van Ginneken, M. Loog, and M.D. Abramoff, "Comparative study of retinal vessel segmentation methods on a new publicly available database", SPIE Med. Imaging, pp. 648- 656, 2004.

[10] Thitiporn Chanwimaluang and Guoliang Fun, "An efficient blood vessel detection algorithm for retinal images using local entropy thresholding." School of Electrical and Computer Engineering, Oklahoma State University, Stillwater, OK 74078

[11] D. Farnell, "ARIA database", [online] available: www.eyecharity.com, 2010. 